\title{
Vinculos entre familia y escuela: visión de los maestros en formación
}

Links Between Family and School: Vision of Teachers in Training

Liens entre famille et école : regard des enseignants en formation

Vínculos entre família e escola: visão dos professores em formação

Fecha de recepción: 21 DE OCTUBRE DE 2014/Fecha de aceptación: 9 DE MAYO DE 2016/Fecha de disponibilidad en línea: 15 DE DICIEMBRE DE 2016

Encuentre este artículo en http://magisinvestigacioneducacion.javeriana.edu.co/

Escrito por Ana CASTRO-ZubizARRETA UnIVERSIDAD DE CANTABRIA, UNICAN

Cantabria, España

castroza@unican.es

Rosa García-Ruiz UnIVERSIDAD dE CANTABRIA, UNICAN

CAnTABria, España rosa.garcia@unican.es

\section{Resumen}

Se presenta la identificación de limitaciones, beneficios y obstáculos en la relación familia y escuela, en España, desde la perspectiva de 134 maestros de educación infantil en formación, por medio de un estudio cualitativo de carácter etnográfico. Los resultados permiten identificar la convivencia en los centros de tres tipos de participación no excluyentes y, aunque con dificultades, se atisba una emergente implicación sistemática de las familias en los planes y proyectos. Esta realidad ha de ser objeto de análisis y reflexión en la formación de maestros, en la que el conocimiento eminentemente teórico se ha de articular con el práctico desde un posicionamiento crítico-reflexivo.

\section{Palabras clave}

Participación de los padres; prácticas de enseñanza; maestros; educación infantil; familia

\section{Transferencia a la práctica}

El estado de la relación familia-escuela es un factor esencial para la mejora de la calidad educativa, entendiendo que el maestro en formación es un agente clave para el futuro cambio educativo si se incide en este contenido durante su formación inicial. La investigación sitúa al maestro en formación en España ante un marco crítico-reflexivo sobre los vínculos familia-escuela percibidos a partir de la realización de prácticas en los centros, como observatorio clave para la reflexión, lo que plantea una propuesta de articulación del contenido eminentemente teórico con el práctico durante la carrera.

Para citar este artículo / To cite this article / Pour citer cet article / Para citar este artigo

Castro-Zubizarreta, A. \& García-Ruiz, R. (2016). Vínculos entre familia y escuela: visión de los maestros en formación. magis, Revista Internacional de Investigación en Educación, 9(18), 193-208. http://dx.doi.org/10.11144/Javeriana.m9-18.vfev 


\section{Keywords}

Parental involvement;

teaching practices; teachers;

early childhood education; family

\section{Mots clés}

Participation des parents; pratiques d'enseignement; enseignants; éducation infantile; famille

\section{Abstract}

The identification of limitations, benefits and obstacles in the family relationship and school in Spain is presented from the perspective of 134 kindergarten teachers in training, through a qualitative ethnographic study. The results allow us to identify the coexistence in schools of three types of inclusive participation and, albeit with difficulty, an emerging systematic involvement of families is clear in the plans and projects. This reality must be the object of analysis and reflection in teacher training, in which the eminently theoretical knowledge has to articulate the practical from a critical-reflexive point of view.

\section{Transfer to practice}

The status of the family-school relationship is essential for improving the quality of education, understanding that teacher training is a key agent for the educational change if this content influences during teachers' initial training. The research puts the teacher in training in Spain before a critical reflective framework about the family-schoo links perceived from the practical work conducted in schools. As such, we propose to articulate the content eminently theoretical with practical approaches during teachers' training.

\section{Résumé}

On présente l'identification de limitations, bénéfices et obstacles dans les rapports de famille et école, en Espagne, depuis la perspective de 134 enseignants d'éducation infantile en formation, au moyen d'une étude qualitative de caractère ethnographique. Les résultats permettent d'identifier le vivre ensemble dans les centres de trois types de participation qui ne sont pas discriminatoires et même s'y il a des difficultés, on entrevoie une émergente implication systématique des familles dans le plans et les projets. II faut que cette réalité soit l'objet d'analyse et réflexion dans la formation des enseignants, dans laquelle la connaissance notamment théorique doit être articulé à la pratique depuis un positionnement critique-réflexive.

\section{Transfert à la pratique}

L'état du rapport famille-école est un facteur essentiel pour améliorer la qualité éducative, en comprenant que l'enseignant en formation est un agent clé pour le futur changement éducatif si l'on affecte dans ce contenu pendant leur formation initiale. La recherche situe à l'enseignant en formation en Espagne en face d'un cadre critique-réflexive par rapport aux liens famille-école perçus à partir de la réalisation de pratiques dans les centres, en tant qu'observatoire clé pour la réflexion, cela offre une proposition d'articulation du contenu notamment théorique avec le pratique pendant la carrière.

\section{Palavras-chave}

Participação dos pais; práticas de ensino; professores; educação infantil; família

\section{Resumo}

Identificam-se limitações, benefícios e obstáculos na relação família e escola, na Espanha, desde a perspectiva de 134 professores de educação infantil em formação, por meio de um estudo qualitativo de caráter etnográfico. Os resultados permitem identificar a convivência de três tipos de participação não excludentes e, ainda que com dificuldades, um emergente e sistemático envolvimento das famílias nos planos e projetos. Esta realidade tem que ser objeto de análise e reflexão na formação de professores, na qual o conhecimento eminentemente teórico deve articular-se com o prático a partir de um posicionamento crítico-reflexivo.

\section{Transferência à prática}

O estado da relação família-escola é um fator essencial na melhora da qualidade educativa e o professor em formação se torna um agente central da mudança educativa esperada, quando este tema é tratado durante sua formação inicial. A pesquisa situa o professor em formação na Espanha num contexto crítico-reflexivo relacionado aos vínculos família-escola, percebidos a partir da realização de práticas nas instituições, como observatório central para a reflexão, o que propõe uma proposta de articulação do conteúdo eminentemente teórico com o prático durante a formação. 


\section{Introducción}

La familia y la escuela se erigen como dos contextos fundamentales en el desarrollo del niño. La investigación educativa recoge ampliamente los beneficios que se desprenden de establecer vínculos fuertes y honestos entre los miembros de la comunidad escolar (Epstein, 1995), que tienen repercusiones positivas para los niños, las familias, los docentes y los centros escolares. Al respecto, la literatura señala mejoras en el rendimiento académico y en el desarrollo personal y social de los niños (Epstein, 2001; Includ-Ed, 2011; Kherroubi, 2008; Mariñas-Gómez \& Rodríguez-Machado, 1999), en su actitud y conducta (Boethel, 2004; Henderson \& Mapp, 2002; Siles-Rojas, 2003). Del mismo modo, se han identificado efectos positivos para las familias que tienden a desarrollar competencias específicas relacionadas con la escuela (Garreta-Bochaca, 2015), afianzando el desarrollo de una parentalidad positiva, así como beneficios para los docentes que sienten mayor implicación con su profesión, incrementan su satisfacción con la misma y mejoran sus habilidades interpersonales (García-Bacete, 2003). Así mismo, estos vínculos repercuten en la mejora de la escuela que se convierte en un espacio más democrático y participativo.

No podemos abordar la temática de este estudio sin definir los constructos participación y relación. Entendemos la relación familia-escuela como el vínculo que se teje entre estas dos instituciones y que, como señalan Núria Llevot y Olga Bernad (2015), pueden desencadenar interacciones positivas o negativas. Con el término participación, nos referimos a la implicación de los progenitores en el centro escolar que se sustenta en una actitud de colaboración que se fomenta si en el centro educativo hay un clima que favorezca en sus integrantes la percepción de sentirse escuchados, respetados y valorados (Castro-Zubizarreta \& García-Ruiz, 2013).

Los estudios que abordan la relación y participación de las familias en el contexto escolar siguen poniendo de relieve que estamos ante una relación compleja (García-Sanz, Gomariz-Vicente, Hernández-Prados \& Parra-Martínez, 2010), pero indispensable. La dificultad de esta relación hace que sea entendida como un desafío (García-Bacete, 2003), en el cual el desencuentro, la distancia, el recelo y el enfrentamiento señalados por Miguel Ángel Santos-Guerra (2000), enfrían y debilitan una relación que debe fraguarse con vocación de servicio al niño.

Para la superación de las dificultades y obstáculos que minan esta relación (Altarejos, 2002; Kñallinsky-Ejdelman, 2003), consideramos imprescindible el planteamiento de marcos de participación en los cuales la escuela sea capaz de canalizar la buena predisposición de las familias para hacerlas partícipes de lo que acontece en el contexto escolar (GarcíaSanz, Gomariz-Vicente, Hernández-Prados \& Parra-Martínez, 2010), pues demandan tener oportunidades de colaboración (Arostegui, Darretxe \& Beloki, 2013; Gallimore \& Goldenberg, 1993; Solé-i-Gallart, 1996).

La participación de las familias en el sistema educativo en el contexto europeo se materializa mediante las asociaciones de madres y padres, y del Consejo Escolar (Egido, 2014). En el panorama normativo español, la participación de las familias está regulada desde 1985 con la Ley Orgánica 8 de 1985, reguladora del derecho a la educación, mediante la participación formal en órganos de representación; en este caso, el consejo escolar del centro. En la actual Ley Orgánica para la Mejora de la Calidad Educativa, LOMCE (España, 2013), se regulan aspectos fundamentales como el derecho a la elección de centro, a la elección del tipo de formación religiosa o moral que prefieren para sus hijos, o al intercambio de información entre familia y escuela, entre otros (Frías del Val, 2014).

\footnotetext{
Descripción del artículo | Article description | Description de l'article | Artigo descrição

Este artículo de investigación, derivado de la investigación La relación familia-escuela desde la mirada del maestro en formación: diagnóstico, análisis y reflexión a través del prácticum de enseñanza, trata de identificar aspectos limitadores y potenciadores de las relaciones entre escuela y familias, a partir de la percepción del profesorado en formación, que lleva a cabo una observación rigurosa durante su período de prácticas en centros educativos españoles. Las conclusiones destacan tres tipos de participación de las familias, y señalan un incremento de esta participación.

Por otro lado, se profundiza en la relevancia del período de prácticas de los profesores en formación, entre los planes de estudio universitarios, para su futuro desarrollo profesional.
} 
Para mejorar y transformar la relación familia-escuela es imprescindible focalizar la mirada en las propuestas de participación que se ofrecen a las familias en el ámbito escolar, en las que coexisten diferentes niveles participativos vinculados con el grado de implicación, la capacidad de decisión y la responsabilidad ampliamente descritos por John Bastiani (1987), Fernando Gil-Villa (1992), Alastair Macbeth (1984) y Carole Pateman (1970), entre otros. Al tomar como referente estas aportaciones, presentamos a continuación una clasificación propia de las modalidades de participación, que a nuestro entender pueden producirse y que concebimos como complementarias y no excluyentes, coincidiendo con Francisco Juan GarcíaBacete (2003), cuando señala que tiene que existir un acuerdo entre familia y escuela en torno al tipo de implicación y participación que desean producir. Estas modalidades son las siguientes:

- Participación informativa o pseudoparticipación, cuando las familias reciben información, asumiendo el rol de espectador, sin capacidad de decisión. El docente es quien toma la iniciativa en este tipo de interacción unidireccional.

- Participación consultiva, en la que las familias forman parte de los órganos de gobierno del centro, como integrantes de la comunidad educativa, representadas en el consejo escolar. Podemos entender esta participación como un nivel de control sobre cuestiones decididas por otros.

- Participación en planes o proyectos del centro, de forma sistemática o esporádica, bien como parte de la Asociación de Madres y Padres del centro, o de manera individual a propuesta del equipo educativo, o por iniciativa propia. Este tipo de participación estaría vinculado con la participación plena en el centro, que otorga a las familias un papel activo en la vida escolar, con capacidad de decisión y reparto de responsabilidades.

Generar marcos de participación efectivos y activos supone conocer las características particulares de cada contexto familiar y escolar, para fraguar relaciones de cordialidad, honestidad y cercanía, que asienten los mimbres de una participación cómoda y asequible, y favorezcan la creación de entornos educativos compartidos (Alguacil-de Nicolás \& Pañellas-Valls, 2009; Comellas, 2006; Lozano-Martínez \& Alcaraz-García, 2013), en los que se valore lo que cada una de las partes puede aportar en el marco de la cultura de la participación (García-Bacete, 2006).

Es preciso, pues, potenciar la colaboración con las familias y reflexionar sobre los canales de participación que se ofertan (Ellis \& Hughes, 2002; Mora, 2002). Ilustrativo nos parece el estudio de Jordi Garreta-Bochaca (2015), quien señala que los canales de comunicación tradicionales como las reuniones de inicio de curso, las tutorías, la agenda escolar, las circulares, las notas a los progenitores y el panel de anuncios siguen siendo los habituales entre la escuela y la familia, y plantea la necesidad de considerar otros canales emergentes como blogs, webs, correo electrónico que, dado su potencial, no pueden ser obviados.

En este proceso de mejora y potenciación de la relación familia-escuela, entendemos que el maestro en formación es un agente clave, motor de cambio, si se incide en este contenido durante su formación inicial, como señalan Francisco Juan García-Bacete (2003) y Eva Kñallinsky-Ejdelman (2003), pues favorece la formación de futuros profesionales que conciban la relación familia-escuela como la oportunidad de compartir la tarea de educar (Ruiz-Corbella, 2007). Todo ello, desde la necesidad de 
articular el contenido eminentemente teórico con el práctico, desde un posicionamiento crítico-reflexivo que ponga de relieve las discordancias y las limitaciones, así como las potencialidades de una relación que el maestro en formación percibe por medio de un observatorio clave para la reflexión: el practicum de enseñanza.

\section{El practicum de enseñanza como momento privilegiado para observar la relación vinculación familia-escuela}

El practicum es un momento clave en la formación de los maestros que tradicionalmente ha favorecido la integración y vertebración de las materias del plan de estudio con la práctica del proceso de enseñanza aprendizaje en el contexto escolar, y que ha cobrado mayor relevancia en la configuración de los planes de estudio de los grados de maestro en el contexto europeo, tras la implantación del nuevo sistema universitario. Destaca, en este nuevo modelo, la función formativa que lo define, y que puede paliar algunas de las deficiencias existentes en la formación universitaria, que señalan Ignacio González-López y Eloísa Reche-Urbano (2010). En este sentido, Miguel Ángel Zabalza-Rebaza (2011) sostiene que complementa los aprendizajes académicos (teóricos y prácticos) con la experiencia en los centros escolares. Por todo ello, coincidimos con María Auxiliadora Chacón-Corzo y Azael Eduardo Chacón-Contreras (2006) cuando se refieren al practicum como un espacio formativo poderoso del ser, saber y saber hacer del futuro docente.

A nuestro entender, el practicum no solo propicia el contacto con la realidad educativa de las aulas, sino que también abre el camino hacia la reflexión sobre la práctica pedagógica. En esta línea se posicionan autores como David A. Kolb (1981), Fritz K. Oser y Franz J. Baeriswyl (2001) y María del Pilar Sepúlveda-Ruiz (2005), entre otros, cuando inciden en la oportunidad que representa el practicum para el análisis de las relaciones existentes entre lo que Mercedes González-Sanmamed y Eduardo José Fuentes-Abeledo (2011) denominan el conocimiento de la enseñanza, conocimiento para la enseñanza y conocimiento en la enseñanza. De este modo, apostamos por un practicum conector de materias que evite la desnaturalización del aprendizaje y lo dote de coherencia y sentido.

Aquí encontramos el mayor reto para el maestro en formación: el proceso de reflexión. Este proceso evita la desconexión (Zeichner, 2010) entre la formación recibida en la universidad y las experiencias generadas durante el practicum de enseñanza. Esa desconexión (Goodlad, 1990; Labaree, 2004; Tejada-Fernández, 2006) ha impulsado un cambio de paradigma que desde los planes de estudio de los grados de maestro plantee el practicum como un lugar de investigación para analizar, codificar, comprender y reflexionar sobre las experiencias de la práctica, siguiendo las ideas de Marilyn Cochran-Smith y Susan L. Lytle (2009), en el sentido de repensar las conexiones entre las materias del plan de estudio y las experiencias de práctica (Zeichner, 2010).

En esta vertiente hay innovadoras experiencias de practicum de maestros que surgen con la pretensión de mejorar el aprendizaje de los maestros en formación, al establecer relaciones teoría-práctica mediante procesos reflexivos (Badia-Pujol, 2012; Brockbank \& McGill, 2002; Domingo, 2010; Gutiérrez-Cuenca, Correa-Gorospe, Jiménez de AberasturiApraiz \& Ibáñez-Etxeberria, 2009), que involucren al alumnado en problemas, desafíos o inquietudes de la educación contemporánea, como plantea en su experiencia Claudia Ximena González-Moreno (2012), para favorecer, en última instancia, que el maestro que finalice su formación 
inicial adquiera y aumente su capacidad de aprender permanentemente de su propia práctica profesional.

En este marco, este trabajo busca acercarnos al estado de la relación familia-escuela, desde la mirada de los maestros de educación infantil en formación, a raíz de su experiencia en el practicum de enseñanza, para facilitar en el estudiante un proceso reflexivo guiado, que intenta favorecer, en la medida de lo posible, la articulación entre la teoría y la experiencia obtenida durante el período de prácticas. De este objetivo general, se desprenden otros objetivos específicos con los que se pretende conocer, a partir de la observación y reflexión de los futuros docentes, los beneficios que se derivan de una adecuada relación familia-escuela, conocer las dificultades o aspectos limitadores que impiden lograr la relación y la participación deseada, e identificar los canales de comunicación más habituales y los tipos de participación presentes en los centros.

\section{Diseño y desarrollo de la investigación}

\section{Participantes}

La muestra está formada por 134 estudiantes del tercer (3) curso del grado de maestro en educación infantil de la Universidad de Cantabria (España), que han realizado sus prácticas en 84 centros educativos de toda la región. La contextualización de las visiones recogidas responde a comunidades educativas diversas, con base en su ubicación en núcleos urbanos y rurales, con titularidad pública, aunque con gestión privada en algunos casos, y que integran diferentes etapas educativas. Todos los tutores de prácticas de estos centros cumplen los criterios de calidad exigidos por la administración educativa (años de experiencia docente, participación en formación continua, responsabilidad como tutor de un grupo-clase). Por lo tanto, se trata de una muestra incidental, cuya participación ha tenido lugar durante el curso 2012-2013.

\section{Metodología de la investigación}

El estudio que presentamos se apoya en un enfoque etnográfico que ha permitido acercar a los maestros en formación a las relaciones que se tejen entre la familia y la escuela. La realización del practicum de enseñanza, durante dos meses en el curso anterior a esta investigación y un mes durante el curso en el que se lleva a cabo la recogida de información, les ha permitido conocer el estado de estas relaciones en los centros escolares en los que han desarrollado una experiencia práctica que favorece los procesos de observación, análisis y reflexión mediante el acercamiento a esta realidad desde una dimensión fundamentalmente práctica.

La técnica seleccionada ha sido la observación participante, entendiendo que los alumnos, durante su período de prácticas de enseñanza, si bien no son observadores expertos, sí tienen acceso a un puesto de observación privilegiado desde donde es posible divisar prácticas y sentidos y resulta, por tanto, una herramienta de investigación que contribuye a la creación de contextos participativos (Rekalde, Vizcarra \& Macazaga, 2014).

\section{Fases del estudio}

- El acceso al ámbito de investigación o escenario: nuestros estudiantes del grado de maestro en educación infantil tienen acceso al ámbito de investigación mediante la realización del practicum de enseñanza. Este hecho favorece que los maestros en formación, como observadores, sean capaces de establecer una buena relación inmediata con los informantes, y recoger datos 
directamente relacionados con los objetivos de la investigación. En este sentido, es necesario señalar que el estudio se nutre de uno de los epígrafes que deben desarrollar en el portafolio de prácticas: agentes educativos, familia y sociedad, y que se vincula al contenido de diferentes materias impartidas a lo largo del grado, entre ellas, contextos didácticos y organizativos de la educación infantil, materia desde la que se orienta el presente estudio y que examina en profundidad la relación familia-escuela con un proceso de sensibilización previo al período de prácticas sobre la pertinencia de su estudio como foco de interés en la formación del futuro docente, así como de análisis y reflexión posterior, sobre el estado de estas relaciones.

- El proceso para realizar la observación y la recogida de datos: la observación por parte del maestro en formación en el centro educativo se realizó siguiendo el proceso descrito por Michael Angrosino y Kimberly Mays de Pérez (2000), que concreta la amplitud de la observación a realizar. De este modo, se llevó a cabo una observación descriptiva por medio del diario de practicum, donde el estudiante observa el entorno y las experiencias que en él se desarrollan asumiendo que lo ignora todo. En segundo lugar, se plantea una observación enfocada a partir de categorías de análisis que apoyaron las decisiones de los estudiantes acerca de lo que deben identificar y recoger de sus diarios. Finalmente, el proceso de observación se concreta mediante la observación selectiva, en busca de respuestas a cuatro grandes interrogantes propuestos que guiaron el proceso de puesta en común y reflexión final sobre la realidad observada y vivencia en el centro escolar. Todo este proceso fue apoyado por el maestro tutor del centro educativo.

- La reflexión teórica: a partir del proceso de observación realizado (gráfico 1) y los datos recogidos, los maestros en formación obtienen una "fotografía" del estado de la relación familia-escuela en su centro de prácticas. Junto a los referentes teóricos, estas herramientas ayudan a explicar y comprender el estado de esta relación, sus potencialidades, limitaciones y propuestas de mejora que estos maestros en formación realizaron desde la observación participante con la expectativa de mejorar su futuro desempeño profesional.

Gráfico 1

Proceso de observación realizado por los maestros en formación

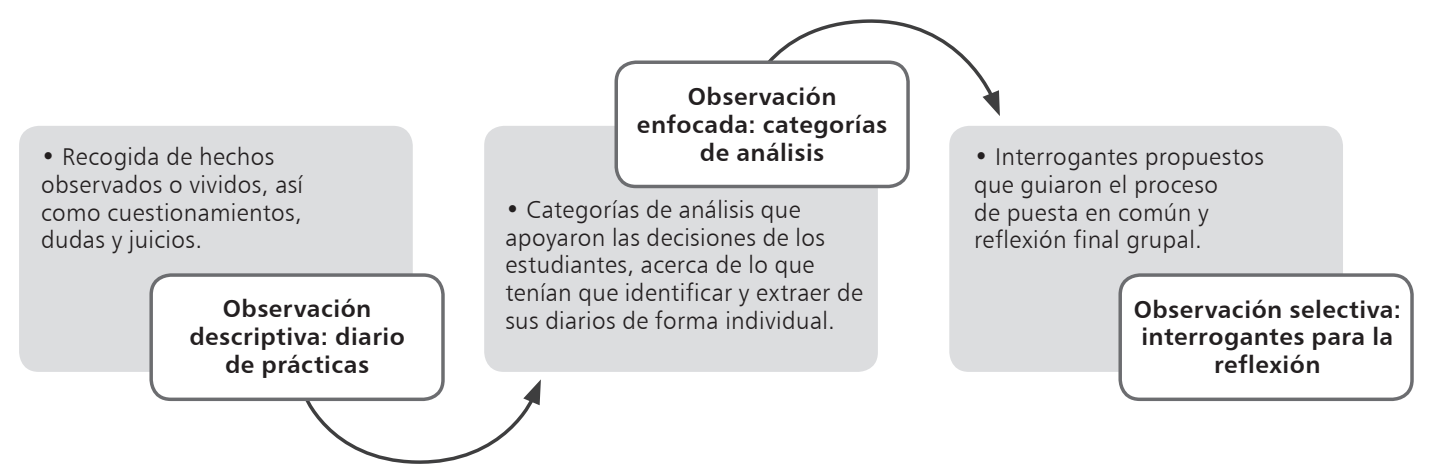

Fuente: elaboración propia 


\section{Instrumentos para la recogida de información}

\section{Diario de prácticas}

El diario se configura como un instrumento que facilita la recogida de datos y la reflexión continuada y sistemática sobre la experiencia vivida en el período de prácticas en el centro escolar, y que forma parte del Portafolio de Prácticas, considerado como herramienta de gran valor orientada hacia la práctica reflexiva, como plantean Beatriz Jarauta-Borrasca y Zoia Bozu (2013). Al respecto, coincidimos con Miguel Ángel Zabalza-Rebaza (2011), cuando señala que el diario es un documento personal en el que se anotan sensaciones, pensamientos y vivencias desde una visión emocionada de la realidad que permitirá al alumnado, a partir de la investigación que presentamos, realizar una lectura diacrónica del diario que favorezca su análisis y la ampliación del conocimiento de los estudiantes sobre la profesión, para comprender y conectar los conocimientos teóricos adquiridos previamente, con la práctica en el aula y la profesión docente.

\section{Categorias de análisis}

Las categorías favorecen la agrupación de información, la concentración de conceptos o temáticas similares. A nuestro entender, son un recurso útil para identificar o señalar temas específicos de un texto, lo que favorece su análisis posterior. Por ello, optamos por aportar al alumnado unas categorías prefijadas que apoyasen las decisiones de los estudiantes acerca de lo que tienen que identificar y extraer de sus diarios de forma individual.

Estas categorías han sido elaboradas a partir del análisis de treinta diarios de prácticas seleccionados al azar de entre los de los 134 estudiantes del grupoclase. En este proceso se realizó una triangulación de investigadores, que partió del análisis del contenido vinculado a la relación familia-escuela en los diarios por cada miembro del equipo investigador de forma independiente, con el que se estableció un sistema de categorías propio para, posteriormente, someterlo a contrastación, utilizando la convergencia de perspectivas para consensuar los hallazgos y definir el sistema de categorías aportado a los maestros en formación y que plasmamos a continuación:

La relación familia-escuela

- Beneficios de la relación familia -escuela

- Dificultades o limitadores de la relación familia - escuela

Marcos de participación familia-escuela

- Canales o medios de transmisión de información

- Tipos de participación
- Participación informativa

- Participación consultiva en órganos de representación del centro

- Participación esporádica o sistemática en planes y proyectos

Interrogantes para la reflexión

Se ofrecen al alumnado interrogantes, preguntas para la reflexión sobre la relación familia-escuela presenciada en el practicum y que buscan respuesta o sustento en el análisis del diario realizado con el sistema de categorías aportado. Los interrogantes planteados fueron los siguientes: ¿qué beneficios se desprenden de la participación de las familias en la escuela?, ¿cómo se relacionan las familias con la escuela?, ¿con quién se establece la comunicación?, ¿qué canales de participación existen en el centro y cuáles se utilizan?, ¿qué aspectos limitadores o dificultades observas en las relaciones entre la escuela y las familias?

El análisis de los datos se realizó con el programa Atlas.ti, en el que se clasificaron los aportes de los alumnos de magisterio en torno a las categorías planteadas con anterioridad y se establecieron subcategorías emergentes derivadas del propio análisis, con su frecuencia de aparición.

\section{Resultados}

El profesorado en formación recoge en su discurso la importancia y necesidad de la relación familia-escuela e identifica beneficios y obstáculos para establecer una adecuada relación entre estos dos agentes educativos. Sin embargo, los beneficios repercuten en mayor medida en los alumnos y en las familias, mientras que no se percibe claramente en qué se beneficia el maestro. Esta visión sorprendente por parte de los maestros en formación coincide con la identificación de docentes a los que no les interesa que se favorezca esta participación, por lo que además de los tradicionales obstáculos que supone la incompatibilidad de horarios con las familias, se detectan actitudes que constituyen una verdadera limitación o barrera a la colaboración y que en la práctica favorece que las familias sean percibidas como extraños o bien que simplemente se les pida colaboración en tareas de índole menor que son entendidas como participación.

\section{Beneficios de la relación familia-escuela:} una relación con la que ganamos todos

Los futuros maestros identifican perfectamente los beneficios de esta relación, como expresa este estudiante:

Los beneficios que se desprenden de esta participación entre otros son: la mejora de la comunicación 
entre escuela y familia, fuente de debate en muchas ocasiones; mejorar el bienestar de los niños en todo momento y conocer lo que este realiza en la escuela.

También señalan que una adecuada relación familia-escuela beneficia sobre todo al alumnado en múltiples aspectos, como el refuerzo de la autoestima, para el $84.3 \%$ de los maestros, su seguridad (88.1\%) y confianza en sus capacidades (81.3\%), del mismo modo que aumenta su iniciativa y participación en el aula (58.5\%), y destacan también para el $75.3 \%$ que una buena relación mejora el rendimiento académico de los alumnos. El término rendimiento académico aparece en las respuestas aportadas por el profesorado en formación, aspecto que evidencia la importancia que confieren a la etapa de educación infantil y su repercusión en etapas educativas ulteriores.

Estos resultados señalan que una óptima relación, que supone un mayor conocimiento y seguimiento del proceso de enseñanza-aprendizaje de los niños por parte de las familias, les reporta múltiples ventajas. Así lo pone de manifiesto una alumna:

Los niños cuyas familias estaban muy conectadas con el centro, desprendían más capacidad participativa y entusiasmo por las actividades en lo concerniente a la narración de actividades externas de fin de semana. Por el contrario, los niños cuyas familias no establecían contacto con el centro, adoptaban una actitud menos entusiasta y participativa, dejando entrever un pequeño pesar ante el claro desvínculo de sus padres con el centro.

Los beneficios que los maestros en formación apuntan en las familias giran en torno al acceso a la información, su participación en el contexto escolar, la generalización de aprendizajes y la coherencia de planteamientos pedagógicos entre el contexto familiar y escolar, así como una mejora en la adaptación al contexto escolar dentro de las aulas de dos y tres años de la etapa de educación infantil. Los resultados de este estudio aportan datos concretos en cuanto a que el $89 \%$ de los maestros en formación considera que las familias se benefician al lograr un mejor seguimiento del proceso de aprendizaje de sus hijos; para el 97\%, la comunicación con el centro mejora, y para el $85.8 \%$, se mejora el acceso a la información. Otros beneficios, aunque en menor medida, son el incremento de sensaciones de seguridad (36.7\%), y la percepción de una mayor competencia en la educación de los hijos (44.7\%). Todo ello en un marco de comunicación que incrementa la seguridad y tranquilidad de las familias y su sentimiento de competencia autopercibida en torno a la educación de sus hijos:

Los padres incrementan el sentido de autoeficacia, mejoran la comunicación con sus hijos en general, valoran más el papel del profesor, están más tranquilos ya que conocen en todo momento cómo progresa su hijo en la escuela.

Como señalamos al principio del epígrafe, los estudiantes de magisterio han priorizado en su discurso las repercusiones positivas de una adecuada relación familia-escuela que identifican tanto en niños como en familias, y han obviado en gran medida los beneficios que aparecen para la acción docente y que debieran, a la luz de los resultados recogidos, ser objeto de análisis y reflexión con el grupo-clase, puesto que tan solo el 5\% de los participantes en el estudio reconocen al colectivo docente como beneficiario de esta relación. A pesar de ello, aparecen aportes que recogen 
el enriquecimiento en la acción educativa que se produce cuando familia y escuela trabajan juntas, como expresan los siguientes estudiantes:

La relación con las familias implica beneficiarse del intercambio de ideas y de información permitiendo a los profesores la oportunidad de enriquecerse y reflexionar, con y sin ellos, sobre las aportaciones de las mismas.

[...] contar con el respaldo de las familias, y con unos alumnos más predispuestos y motivados hacia el aprendizaje, provocará que su labor se vea enriquecida al igual que su motivación a la hora de llevar a cabo la docencia.

\section{Dificultades observadas, nadie dijo que fuera fácil: de las interferencias} en la comunicación, a los desencuentros y malentendidos

Los estudiantes señalan la existencia en la etapa de educación infantil de unas características metodológico-organizativas que debieran favorecer la relación y el hecho de que esta etapa sea el primer contexto escolar con la que familia y niño tienen contacto. Así lo constata una alumna:

Pienso que, en general, la educación infantil es una etapa especialmente propicia para que la interrelación de ambos agentes sea positiva, pues existe mayor disposición de los familiares (suele ir mermando según cumplen años) y la estructura escolar, sin una división tan rígida de horarios y asignaturas, también parece favorecerlo.

A pesar de este marco favorecedor de la relación, el 93\% de los maestros en formación señala limitaciones como el horario laboral de las familias y su incompatibilidad con el horario escolar (73.1\%), la poca comunicación existente (72.3\%), los sentimientos de incompetencia e inseguridad en las familias en relación con la participación (26\%), o la idea de que solo el tutor convoca a las familias para hablar de algún problema presente en el niño que, entre otras, condicionan lo que podría entenderse como una relación deseable (23\%). En este sentido, la falta de comunicación o de contacto por incompatibilidades horarias se erige como uno de los obstáculos más importantes por superar, como constatan los maestros en formación:

En algún caso específico, los horarios de trabajo, [...] como he podido comprobar el contacto físico con alguna familia no se llega a realizar por la falta de tiempo.

Pero sugieren que esta limitación puede entenderse como un elemento fácilmente subsanable, si se contase con una actitud que favoreciese la relación. Al respecto, los maestros en formación señalan que una adecuada relación familia-escuela se sustenta en una actitud que favorezca la escucha, el encuentro y la participación, e infieren que debe darse en primer lugar en los maestros, concibiéndolos como sus iniciadores y dinamizadores.

Un aspecto a considerar es cómo los maestros en formación señalan que son los propios docentes los que limitan la relación y participación de las familias en el centro, pues su actitud es el foco del distanciamiento:

Sin embargo, pienso que el principal escollo para la implicación de las familias en la escuela son los propios docentes. Muchos ven a los padres de sus alumnos como estorbos a los que mantener alejados del aula o como simples receptores de información. 
A pesar de que estos aportes deben ser considerados con cautela, ya que no se ofrece una triangulación de perspectivas, no podemos obviar que los maestros en formación señalan encontrarse con maestros que evitan la relación con las familias, al limitar su contacto a lo legal y organizativamente estipulado.

Por otro lado, los maestros en formación coinciden en señalar que las familias se interesan por lo que sus hijos realizan en el ámbito escolar, si bien identifican desencuentros entre maestros y familias en torno a expectativas en relación con sus hijos:

En mi caso, la tutora tuvo una reunión con una familia sobre la evolución de su hijo y [los padres] no aceptaban nada de lo que la profesora les decía; a las responsabilidades en torno a su educación: los docentes culpan a las familias de las dificultades de los niños, las critican por no ayudar a los niños en la realización de la tarea... Mi tutora se quejaba de la delegación de funciones, de que cada vez se les pide [sic] más responsabilidades y tienen menos apoyo.

Y la coherencia pedagógica entre el contexto familiar y escolar:

Mi tutora de prácticas comentando con ella un caso de un niño me dijo que, a veces, todo lo que avanzaba entre semana se perdía en dos días del fin de semana en casa.

\section{Una fotografía de la participación en los centros}

A partir de las reflexiones que los estudiantes plasman en sus diarios de prácticas y de los interrogantes planteados, podemos identificar los tres tipos de participación que se han presentado en la fundamentación de este trabajo además de identificar los canales de comunicación más habituales.

Los estudiantes de magisterio señalan que los canales de comunicación más utilizados para el intercambio de información son los usados tradicionalmente, lo que se evidencia por el $96.2 \%$ de los participantes en este estudio, siendo estos el envío de notas, la comunicación telefónica, los paneles informativos a la entrada de la clase y las reuniones tanto grupales como individuales con el maestro tutor. De esta forma, lo expresa esta estudiante:

Durante las prácticas, percibí que los niños llevaban notas tanto del profesor como del director y la profesora tenía reuniones con los padres todas las semanas, cada semana solía tener a dos familias.

Del mismo modo, el $74.6 \%$ de los maestros en formación comenta cómo las familias aprovechan las entradas y las salidas de clase para intercambiar información sobre la evolución de su hijo.

En todo caso, el $64.2 \%$ de los maestros pone de manifiesto el incremento de las plataformas digitales, como blogs, o webs, por parte del profesorado de educación infantil como medio de comunicación eficaz con las familias, con las que se comparte la vida cotidiana del aula. Así mismo, los maestros en formación señalan su utilidad y perciben que, a pesar de que su dinamización por parte del profesorado implicado suponga un gran esfuerzo, estas plataformas pueden ser un recurso rico de intercambio de información entre compañeros docentes, de formación permanente e innovación pedagógica. Así lo refleja esta estudiante:

En mi centro contaban con un blog de las dos clases de infantil que iba modificándose con continuidad y en el que la participación de los padres era totalmente abierta. Estaba genial y facilitaba mucho la comunicación y la participación de las familias, que esperaban con ganas nuevas entradas en el blog. [...] Mi tutora en el centro decía que sentía recompensadas las horas que pasaba en su casa frente al ordenador actualizando el blog por los mensajes de las familias y que solía visitar blogs de otros compañeros para coger ideas.

Este tipo de participación informativa o pseudoparticipación de las familias está presente en la totalidad de los centros escolares en los que se ha contextualizado el estudio, según el $78.3 \%$ de los participantes; si bien, hay una mayor diversidad en los centros si se contempla una participación con un mayor nivel de implicación y responsabilidad en las familias. En relación con la participación consultiva en órganos de representación del centro, hemos de señalar que si bien los alumnos de magisterio son conocedores de la existencia y funcionamiento del consejo escolar, el $80.9 \%$ de ellos manifiesta no haber podido observar su funcionamiento durante el practicum de una manera directa, pero sí han obtenido información por medio de los tutores de los centros. Al igual sucede con el funcionamiento del AMPA (Asociación de Madres y Padres de Alumnos) y concluyen que la participación de las familias en estos órganos es escasa, pero destacan la gestión y dinamización de la participación de las familias en la escuela mediante su relación con el maestro-tutor. De esta forma lo expresaba esta estudiante:

Lo que yo he percibido es que la participación por parte de las familias se establece directamente con los tutores, ya que es con los que más trato tienen. 
En este sentido, el $90.3 \%$ de los maestros en formación dice haber percibido una participación esporádica de las familias en los centros, es decir, participan en momentos puntuales, a petición del maestro-tutor, colaboran en festividades, fechas señaladas y puntuales y que por lo tanto, no entran en la vida cotidiana de las aulas. Ejemplo de esta realidad es la vivencia que nos transmite esta alumna:

Hay padres que acuden al centro en ocasiones excepcionales a colaborar, estas ocasiones son por ejemplo: a realizar disfraces para carnavales, a hacer bocadillos el día de la comida solidaria, etc. Pero nunca he sido partícipe de que los padres hayan acudido al centro para participar de la educación de sus hijos mediante talleres $\mathrm{u}$ otras propuestas.

En todo caso, atisbamos en el discurso de los maestros en formación la aparición de una emergente implicación sistemática, continuada en el tiempo, de las familias en los centros por medio de su participación en planes y proyectos, lo que supone un nivel superior de participación y presencia de las familias en la escuela que se sustenta en una actitud colaborativa y de disponibilidad, tanto por maestros como por familias, favorecida principalmente por la realización de talleres, según el 17\% de los maestros en formación, y por la participación en la metodología basada en proyectos en el aula, para el 29,8\% de los maestros formación. El comentario de esta estudiante nos parece ilustrativo:

Las familias colaboran casi a diario con la escuela pues la metodología de esta está basada en proyectos por lo que las familias investigan con el niño, juntos desde casa para luego compartirlo en clase. Por otra parte, cada jueves en este colegio es día de talleres conjuntos, en los que se crean grupos de alumnos de diferentes edades y en los que aquel padre, madre, abuelo o familiar que quiera y pueda participar, será bienvenido durante toda la jornada escolar. Finalmente, otra canal que he podido observar ha sido el acompañamiento en las salidas programadas por el colegio a la cual puede asistir quien quiera.

\section{Conclusiones y discusión}

Los maestros en formación se refieren a la etapa de educación infantil como un marco favorecedor de la relación familia-escuela, dadas las características metodológico-organizativas que presenta, pues las familias están dispuestas a participar, deseosas de conocer la adaptación y desarrollo de su hijo en el contexto escolar. La participación observada por los maestros en formación ofrece una fotografía en la cual predomina la pseudoparticipación o participación meramente informativa de las familias que, en menor medida, coexiste con una participación consultiva vinculada con la representación de las familias en los órganos de gobierno escolar de los centros. Del mismo modo, aparece en el discurso del maestro en formación la vivencia de escasas manifestaciones de la participación en planes o proyectos de las familias, si bien la entendemos como una tendencia emergente en los centros que puede derivar hacia una participación familiar que suponga un mayor grado de continuidad y responsabilidad de su parte en el centro escolar. Coincidimos por tanto, con Francisco Juan García-Bacete (2003), cuando destaca el potencial de los proyectos que van naciendo en los centros para dinamizar la participación de las familias, y de los nuevos canales online, principalmente los blogs de aula que facilitan la comunicación (Garreta-Bochaca, 2015). Así mismo, los resultados obtenidos indican que la totalidad de los maestros en formación señala haber observado los beneficios que se desprenden de una adecuada relación familia-escuela. Así mismo, un elevado porcentaje considera que las familias se benefician al lograr un mejor seguimiento del proceso de aprendizaje de sus hijos, lo que mejora la comunicación con el centro y el acceso a la información. Otros beneficios que se encuentran, aunque en menor medida, son el incremento de sensaciones de seguridad y la percepción de una mayor competencia, habilidad en la educación de los hijos. Pero el principal beneficiario de esta adecuada relación es sin duda el alumno: la mayoría de los participantes destaca el aumento de su motivación, la sensación de seguridad y la mejora del rendimiento académico y, en menor medida, el aumento de la participación en actividades del aula como principales beneficios. Respecto a lo que esta relación reporta en el colectivo docente, la mayoría de los participantes en este estudio no reconoce al maestro como beneficiario, por lo que entendemos que se trata de un pensamiento discordante del futuro docente, identificado a raíz de este trabajo y sobre el que se precisa reflexionar y redefinir durante su proceso formativo. La discusión de los resultados con los señalados en investigaciones previas, permite constatar que la incompatibilidad horaria es uno de los principales obstáculos (Altarejos, 2002; CastroZubizarreta \& García-Ruiz, 2013; Kñallinsky-Ejdelman, 2003). Pero emergen otros obstáculos, derivados de la actitud de algunos docentes que prefieren evitar la participación de las familias, en ocasiones, para no tener desencuentros con algunos padres respecto a las expectativas sobre sus hijos, y se evidencia una falta de comprensión o entendimiento mutuo, que incide de forma negativa en la relación familia-escuela y la participación de esta en el contexto escolar. En esta línea se manifiesta Jordi Garreta-Bochaca (2015), que 
hace referencia a que los maestros creen que la familia debe implicarse en "su justa medida", pero esa medida está condicionada por un matiz subjetivo que dificulta claramente el acuerdo.

Así mismo, consideramos que un obstáculo preocupante es la existencia de resistencias del profesorado en torno a la participación de la familia, ya que entendemos su figura como clave en la promoción de una adecuada relación. Esta situación es recogida por el informe del Consejo Escolar del Estado (2014), que advierte sobre la creencia de algunos profesores de que las familias y su participación en el centro escolar suponen una interferencia en su trabajo, lo ralentiza o interrumpe y así ha sido observado también por los estudiantes. Este hecho tiene su origen — desde la visión de Marta Ruiz-Corbella (2007) — en la falta de formación del profesorado en el campo de la educación familiar, que esta autora percibe como una asignatura pendiente del profesorado que conlleva desencuentros con las familias.

De este discurso se concluye que la relación con las familias continuará generando tensiones entre los docentes en la medida en que no se superen las mencionadas actitudes de bloqueo o de rechazo a la participación de las familias. En este sentido, la contradicción entre la formación teórica de los maestros que enfatiza en la importancia de establecer unos vínculos sólidos, verdaderos y permanentes con las familias, se hace evidente cuando los futuros maestros constatan que en su práctica profesional, los maestros no han superado los obstáculos que impiden esa relación ideal, incluso son ellos los que en ocasiones no desean superarlos.

Estos resultados requieren nuevas líneas de investigación que ayuden a comprenderlos. En este estudio se destaca también la necesidad de profundizar en la formación inicial de los maestros, con la intención de sensibilizar a los docentes hacia la superación de barreras u obstáculos que se puedan presentar en las relaciones con las familias, lo que vincula su formación práctica en los centros educativos con la necesidad de generar una actitud crítica y reflexiva, que fomente su papel responsable como impulsores y facilitadores de la implicación y participación activa de las familias. Por ello, es preciso dar a conocer a los maestros en formación los centros educativos que están teniendo buenos resultados en la mayor implicación de las familias en el proceso.

Las conclusiones derivadas de esta investigación impulsan nuevas responsabilidades, relacionadas con la formación inicial del profesorado que, sin duda, deben superar algunas de las limitaciones encontradas, como pueden ser las características de la muestra, o la ampliación de la recogida de información a los docentes en activo y las familias, para llegar a diseñar, a partir del conocimiento de la realidad, entornos educativos compartidos, basándonos en la reflexión de los maestros, como profesionales autónomos y dinamizadores de la participación de la comunidad educativa, en el sentido que señala Marta Ruiz-Corbella (2007), lo que incide en la necesidad de articular la teoría abordada en las asignaturas de su carrera con la práctica vivenciada en los centros educativos, tomando, en nuestro caso, como eje vertebrador, el practicum de enseñanza.

\section{Sobre las autoras}

Ana Castro-Zubizarreta es profesora del departamento de educación, Universidad de Cantabria, adscrita al área de teoría e historia de la educación. Líneas de investigación: educación infantil, formación permanente del profesorado, transición educativa, procesos de escucha y participación infantil.

Rosa García-Ruiz es profesora del departamento de educación, Universidad de Cantabria, adscrita al área de didáctica y organización escolar. Líneas de investigación: calidad educativa, alfabetización mediática, formación permanente, metodología docente.

\section{Referencias}

Alguacil-de Nicolás, M. \& Pañellas-Valls, M. (2009). Implicación de las familias en los institutos de enseñanza secundarias. magis, Revista Internacional de Investigación en Educación, 2(3), 111-128. Recuperado de http://revistas.javeriana.edu.co/ index.php/MAGIS/article/view/3403/2590

Altarejos, F. (2002). La relación familia-escuela. Estudios sobre Educación, ESE, 3, 113-120. Recuperado de http://dadun.unav.edu/bitstream/10171/8235/1/ Nb.pdf

Angrosino, M. V. \& Mays de Pérez, K. A. (2000). Rethinking Observation: From Method to Context. En Norman K. Denzin \& Yvonna S. Lincoln (eds.). Handbook of Qualitative Research, 673702. Thousand Oaks, California: Sage.

Arostegui, I.; Darretxe, J. \& Beloki, N. (2013). La participación de las familias y de otros miembros de la comunidad como estrategia de éxito en las escuelas. Revista Iberoamericana de Evaluación Educativa, 6(2), 187-200. Recuperado de http:// www.rinace.net/riee/numeros/vol6-num2/ art10.pdf

Badia-Pujol, J. (2012). Ayudar al desarrollo profesional de los docentes: www.practicareflexiva.pro. magis, Revista Internacional de Investigación en Educación, 4(9), 757-764. Recuperado de http:// revistas.javeriana.edu.co/index.php/MAGIS/ article/viewFile/3587/2701 
Bastiani, J. (1987). From Compensation... to Participation? A Brief Analysis of Changing Attitudes in the Study and Practice on Home-School Relation. En John Bastiani (ed.). Parents and Teachers. Perspectives on Home-School Relations, 88-107. Windsor, Berkshire, England: National Foundation for Educational Research, NFER-Nelson (Granada Learning Assessment).

Boethel, M. (2004). Readiness: School, Family, and Community Connections. Austin, Texas: Southwest Educational Development Laboratory, SEDL. Recuperado de http://www.sedl.org/ connections/resources/readiness-synthesis.pdf

Brockbank, A. \& McGill, I. (2002). Aprendizaje reflexivo en la educación superior. Madrid: Morata.

Castro-Zubizarreta, A. \& García-Ruiz, R. (2013). La visión del profesorado de educación infantil y primaria de Cantabria sobre la participación y las relaciones interpersonales entre los miembros de la comunidad escolar. Aula Abierta, 41(1), 73-84. Recuperado de https://dialnet.unirioja.es/descarga/ articulo/4097758.pdf

Chacón-Corzo, M. \& Chacón-Contreras, A. (2006). Los diarios de prácticas: una estrategia de reflexión en la formación docente. Acción Pedagógica, 15, 20-127. Recuperado de https://dialnet.unirioja. es/descarga/articulo/2968965.pdf

Cochran-Smith, M. \& Lytle, S. L. (2009). Inquiry as Stance: Practitioner Research for the Next Generation. New York: Teachers College Press.

Comellas, M. J. (2006). Nuevas alternativas y modelos en la relación familia-escuela y profesionales de la comunidad. Cultura y Educación, 18(3-4), 295-310. Recuperado de http://www.grode. org/admin/index.php?pagina=descargar\&d $o c=1446444360-0-3 . p d f$

Domingo, À. (2010). La práctica reflexiva en los estudios de Magisterio de la UIC. En Olga Esteve, Ko Melief \& Ángel Alsina (eds.). Creando mi profesión. Una propuesta para el desarrollo profesional del profesorado. Octaedro: Barcelona.

Egido, I. (2014). Marcos normativos de la participación de las familias en los sistemas educativos europeos. Una visión comparada. En La participación de las familias en la educación escolar, 35-56. Madrid: Ministerio de Educación, Cultura y Deporte, Secretaría General Técnica. Recuperado de http:// www.mecd.gob.es/dctm/cee/publicaciones/ estudioparticipacion/estudioparticipacion. pdf?documentld=0901e72b81b45e35

Ellis, D. \& Hughes, K. (2002). Connecting Schools, Families, and Communities for Youth Success. Partnerships by Design. Cultivating Effective and Meaningful School-Family-Community Partnerships. Portland, Oregon: Northwest Regional
Educational Laboratory. Recuperado de http:// educationnorthwest.org/sites/default/files/ pbd.pdf

Epstein, J. L. (1995). School/family/community partnerships: Caring for the children we share. Phi Delta Kappan, 76(9), 701-712.

Epstein, J. L. (2001). School, Family, and Community Partnerships. Preparing Educators and Improving Schools. Boulder, Colorado: Westview Press.

España, Consejo Escolar del Estado (2014). La participación de las familias en la educación escolar. Madrid: Ministerio de Educación, Cultura y Deporte. Recuperado de http://www.mecd.gob.es/dctm/cee/ publicaciones/estudioparticipacion/estudioparti cipacion.pdf?documentld=0901e72b81b45e35

España (2013). Ley Orgánica 8/2013, 9 de diciembre, para la mejora de la calidad educativa, LOMCE. Boletín Oficial del Estado, BOE, 295, 10 de diciembre de 2013. Recuperado de https://www.boe. es/diario_boe/txt.php?id=BOE-A-2013-12886

Frías del Val, A. S. (2014). Evolución del marco normativo español sobre la participación de las familias. Contexto histórico. En La participación de las familias en la educación escolar, 57-80. Madrid: Ministerio de Educación, Cultura y Deporte, Secretaría General Técnica. Recuperado de http://www.mecd.gob.es/dctm/cee/publicaciones/estudioparticipacion/estudioparticipacion. pdf?documentld=0901e72b81b45e35

Gallimore, R. \& Goldenberg, C. (1993). Activity Settings of Early Literacy: Home and School Factors in Children's Emergent Literacy. En Ellice A. Forman, Norris Minick \& C. Addisson Stone (eds.). Context for Learning. Sociocultural Dynamics in Children's Development, 314-335. New York: Oxford University Press.

García-Bacete, F. J. (2003). Las relaciones escuela-familia: un reto educativo. Infancia y Aprendizaje, 26(4), 425-437. Recuperado de Inx.educacionenmalaga.es/valores/files/2011/12/Relacionesescuela-familia1.pdf

García-Bacete, F. J. (2006). Cómo son y cómo podrían ser las relaciones entre escuelas y familias en opinión del profesorado. Cultura y Educación: Revista de Teoría, Investigación y Práctica, 18(3-4), 247-266.

García-Sanz, M. P.; Gomariz-Vicente, M. A.; Hernández-Prados, M. A. \& Parra-Martínez, J. (2010). La comunicación entre la familia y el centro educativo, desde la percepción de los padres y madres de los alumnos. Educatio Siglo XXI, 28(1), 157188. Recuperado de http://revistas.um.es/edu catio/article/view/109771/104461

Garreta-Bochaca, J. (2015). La comunicación familiaescuela en educación infantil y primaria. Revista 
de la Asociación de Sociología de la Educación, RASE, 8 (1), 7185. Recuperado de https://www.researchgate.net/publication/ 283071318_Garreta_J_La_comunicacion_familia-escuela_en_ educacion_infantil_y_primaria_Revista_de_la_Asociacion_de_ Sociologia_de_la_Educacion_RASE_n_8_vol_1_pp_71-85_2015

Gil-Villa, F. (1992). La participación democrática en los centros de enseñanza no universitarios. Madrid: Centro de Investigación y Documentación Educativa, CIDE.

González-López, I. \& Reche-Urbano, E. (2010). Las demandas formativas del alumnado de magisterio. Construcción de un plan de formación complementaria. magis, Revista Internacional de Investigación en Educación, 2(4), 383-400. Recuperado de http://revistas.javeriana. edu.co/index.php/MAGIS/article/view/3520/2612

González-Moreno, C. X. (2012). Formación del pensamiento reflexivo en estudiantes universitarios. magis, Revista Internacional de Investigación en Educación, 4(9), 595-617. Recuperado de http://revistas. javeriana.edu.co/index.php/MAGIS/article/download/3578/2692

González-Sanmamed, M. \& Fuentes-Abeledo, E. J. (2011). El practicum en el aprendizaje de la profesión docente. Revista de Educación, 354, 47-70. Recuperado de www.revistaeducacion.educacion.es/re354/ re354 03.pdf

Goodlad, J. (1990). Connecting the Present to the Past. En John I. Goodlad, Roger Soder \& Kenneth A. Sirotnik (eds.). Places Where Teachers are Taught, 3-39. San Francisco, California: Jossey-Bass.

Gutiérrez-Cuenca, L.; Correa-Gorospe, J. M.; Jiménez de Aberasturi-Apraiz, E. \& Ibáñez-Etxeberria, A. (2009). El modelo reflexivo en la formación de maestros y el pensamiento narrativo: estudio de un caso de innovación educativa en el Practicum de Magisterio. Revista de Educación, 350, 493-505. Recuperado de http://www.ince.mec.es/ revistaeducacion/re350/re350_22.pdf

Henderson, A. T. \& Mapp, K. L. (2002). A New Wave of Evidence. The Impact of School, Family, and Community Connections on Student Achievement. Austin, Texas: Southwest Educational Development Laboratory, SEDL. Recuperado de https://www.sedl.org/connections/ resources/evidence.pdf

Includ-Ed, Strategies for Inclusion and Social Cohesion in Europe from Education (2011). Integrated Project Priority 7 of Sixth Framework Programme, 2006-2011. Recuperado de http://creaub.info/included/ about/

Jarauta-Borrasca, B. \& Bozu, Z. (2013). Portafolio docente y formación pedagógica inicial del profesorado universitario. Un estudio cualitativo en la Universidad de Barcelona. Educación XX1, 16(2), 343-362. Recuperado de http://diposit.ub.edu/dspace/bits tream/2445/65191/1/609588.pdf. DOI: 10.5944/educxx1.16.2.2646

Kherroubi, M. (dir.) (2008). Des parents dans l'école. Ramonville Saint-Agne: Éditions Érès.

Kñallinsky-Ejdelman, E. (2003). Familia-Escuela: una relación conflictiva. El Guiniguada, 12, 71-94. Recuperado de http://repositorio.ulpgc.es/ bitstream/10553/5469/1/0235347_02003_0007.pdf

Kolb, D. A. (1981). Learning Styles and Disciplinary Differences. En Arthur W. Chickering \& Associates. The Modern American College: Responding to the New Realities of Diverse Students and a Changing Society, 232-255. San Francisco, California: Jossey-Bass. Recuperado de http://learningfromexperience.com/media/2010/08/Learning-stylesand-disciplinary-difference.pdf 
Labaree, D. (2004). The Trouble with Ed Schools. New Haven: Yale University Press.

Llevot, N \& Bernad, O. (2015). La participación de las familias en la escuela: factores clave. Revista de la Asociación de Sociología de la Educación, 8(1), 57-70. Recuperado de http://www.ase.es/rase/ index.php/RASE/article/view/370/371

Lozano-Martínez, J. \& Alcaraz-García, S. (2013). Los centros educativos multiculturales y sus relaciones con las familias: el caso de la región de Murcia. Educación XX1, 16(1), 210-232. Recuperado de http://revistas.uned.es/index.php/ educacionXX1/article/viewFile/724/2501. DOI: 10.5944/educxx1.16.1.724

Macbeth, A. (1989). Involving Parents: Effective Parent-Teacher Relations. Oxford: Heinemann Educational Books.

Mariñas-Gómez, M. J. \& Rodríguez-Machado, E. (1999). Relación familia-centro escolar. Propuestas de participación. Revista Galego-Portuguesa de Psicoloxía e Educación: Revista de Estudios e Investigación en Psicología y Educación, REI$P E, 4$, 55-68. Recuperado de http://ruc.udc.es/ dspace/bitstream/handle/2183/6687/RGP_4-4. pdf? sequence $=1$ \&is Allowed $=y$

Mora, J. C. (2002). Canales de comunicación familiaescuela. Infancia: educar de 0 a 6 años, 74, 10-14.

Oser, F. K. \& Baeriswyl, F. J. (2001). Choreographies of Teaching: Bridging Instruction to Learning. En Virginia Richardson (ed.). Handbook of Research on Teaching, 1031-1065. Washington: American Educational Research Association, AERA.

Pateman, C. (1970). Participation and Democratic Theory. London: Cambridge University Press.

Rekalde, I.; Vizcarra, M. T. \& Macazaga, A. M. (2014). La observación como estrategia de investigación para construir contextos de aprendizaje y fomentar procesos participativos. Educación XXI, 17(1), 199-220. Recuperado de http://revistas. uned.es/index. php/educacionXX1/article/viewFile/10711/11305. DOI: 10.5944/educxx1.17.1.1074
Ruiz-Corbella, M. (2007). La participación, vía de encuentro entre padres y centro educativo. Participación Educativa, 4, 54-59. Recuperado de https://sede.educacion.gob.es/publiventa/detalle.action?cod=14197

Santos-Guerra, M. Á. (2000). La participación es un árbol. Padres y madres, desde la ciudadanía, hacen la escuela. Kirikiki. Cooperación Educativa, 55-56, 105-116.

Sepúlveda-Ruiz, M. P. (2005). Las prácticas de enseñanza en el proceso de construcción del conocimiento profesional. Revista Educar, 36, 71-93. Recuperado de www.raco.cat/index.php/Educar/ article/download/39743/39581

Siles-Rojas, C. (2003). La colaboración de los padres con la escuela. Revista Padres y Maestros, P\&M, 279, 10-14. Recuperado de http://revistas.up comillas.es/index.php/padresymaestros/article/ view/2020/1753

Solé-i-Gallart, I. (1996). Las relaciones entre familia y escuela. Cultura y Educación: Revista de Teoría, Investigación y Práctica, 4, 11-17.

Tejada-Fernández, J. (2006). El practicum por competencias: implicaciones metodológico-organizativas y evaluativas. Bordón, 58(3), 403-422. Recuperado de http://recyt.fecyt.es/index.php/ BORDON/issue/view/2276

Zabalza-Beraza, M. Á. (2011). El practicum como socialización profesional. Organización y Gestión Educativa: Revista del Fórum Europeo de Administradores de la Educación, 6(19), 11-13.

Zeichner, K. (2010). Nuevas epistemologías en la formación del profesorado. Repensando las conexiones entre las asignaturas del campus y las experiencias de prácticas en la formación del profesorado en la universidad. Revista Interuniversitaria de Formación del Profesorado, 68(24.2), 123-149. Recuperado de http://www.aufop.com/aufop/ uploaded_files/articulos/1279237815.pdf 